\title{
sciendo
}

\author{
BULGARIAN ACADEMY OF SCIENCES
}

CYBERNETICS AND INFORMATION TECHNOLOGIES • Volume 18, No 3

Sofia $2018 \quad$ Print ISSN: 1311-9702; Online ISSN: 1314-4081

DOI: $10.2478 /$ cait-2018-0040

\section{Multi-Layer Cluster Based Energy Aware Routing Protocol for Internet of Things}

\author{
S. Sankar ${ }^{1}$, P. Srinivasan ${ }^{2}$ \\ ${ }^{1}$ School of Computer Science and Engineering, VIT University, Vellore-632014, Tamilnadu, India \\ ${ }^{2}$ School of Information Technology and Engineering, VIT University, Vellore-632014, Tamilnadu, India \\ E-mails: sankar.s@vit.ac.in_srinivasan.suriya@vit.ac.in
}

Abstract: We propose a multi-layer cluster based energy aware routing protocol for Low Power and Lossy Networks, which divides the network area into equal length rings. The intra-ring clustering process divides a ring into equal sized clusters and inter-cluster routing applies the fuzzy logic to select the best route for data transfer. It increases the network lifetime and packet delivery ratio by $18-22 \%$ and 5-8\%, respectively.

Keywords: Internet of things, low power and lossy networks, IPv6 routing protocol for low power and lossy networks, cluster head, cluster member.

\section{Introduction}

Internet of Things (IoT) is an emerging research area in Information and Communication Technology (ICT). IoT provides various solutions to the problems in different domains [1]. It is a collection of sensor-enabled physical objects connected to the Internet, which exchanges data between them without human involvement. Its applications are vital in the smart home, smart grid, smart city, smart agriculture, building automation, etc., [6, 16].

The Low power and Lossy Network (LLN) contain highly resource-constrained wireless devices, which have low processing capacity and transmission rate [17]. IEEE 802.16 is quite lossy compared to IEEE 802.11 [21]. IPv6 Routing Protocol for Low power and lossy networks (RPL) is the standardised routing protocol for LLN by IETF [22]. It is a distance vector and source routing protocol designed explicitly for LLN. It follows the Destination-Oriented Directed Acyclic Graph (DODAG) like tree topology. The DODAG root is connected directly to the Internet. It generates RPL instances, each RPL instance may contain more than one DODAG, uniquely identified by DODAGID. The source node transfers the data to the destination or receiver node via DODAG root. Upward routing indicates the edge directed towards 
the DODAG root and downward route indicates the edge direction far away from the DODAG root [3].

Although RPL fulfils the requirements of LLN, we require a lot improvement in RPL to prolong the network lifespan and to provide the Quality of Service (QoS). Network data traffic, load imbalance problem in an uneven parent selection process, multi-sink and multi-instance problem in mobility scenario, interoperability issues in vast Internet hosts, link failure and local repair in parent unreachable situation, fault tolerance and security mechanism are the major challenges in RPL [4].

The major contribution of this paper is to address the problem of multipoint-topoint (MP2P) data traffic, to extend the network lifetime. In this paper, we propose a Multi-layer Cluster based Energy Aware Routing Protocol (MCEA-RPL) for LLN. In MCEA-RPL, the network area is divided into rings of equal width, using the finest ring width for the network space. In the intra-ring clustering process, it forms equalsized clusters in a ring. In inter-cluster routing, the parent selection process applies the fuzzy logic over the routing metric Expected Transmission count (ETX) and Residual Energy (RER), to select the optimal parent node, for transferring the data efficiently.

The paper is organized as follows: Section 2 describes the related work. Section 3 represents the MCEA-RPL protocol. Section 4 discusses the result and discussions. Section 5 is the conclusion and future work of this paper.

\section{Related work}

In this section, we discuss the cluster based RPL, ring topology based cluster routing model and fuzzy logic based clustering protocol in WSN.

T a n [20] proposed the cluster based RPL protocol to prolong the network lifetime in LLN. It splits the network area into layers and each layer is considered as a cluster. Each cluster selects the $\mathrm{CH}$ node based on the remaining energy. All the Cluster Members $(\mathrm{CM})$ forward the data to Cluster Head $(\mathrm{CH})$. The $\mathrm{CH}$ node aggregates and forwards the data to DODAG root. $\mathrm{Zh} \mathrm{ang}$ et al. [24] proposed an energy Efficient Heterogeneous Ring Clustering protocol (E2HRC) to extend the network lifetime of WSN. It forms rings of equal area in the network space and it selects the $\mathrm{CH}$ node rotationally. The $\mathrm{CM}$ sends the data packets to the $\mathrm{CH}$ node. The $\mathrm{CH}$ node aggregates and forwards the data packets to parent $\mathrm{CH}$. Likewise, the data is forwarded to DODAG root. $\mathrm{Zang}$ et al. [25] proposed an Improved RPL (IRPL) for WSN. It divides the network area into equal size of rings. It selects the $\mathrm{CH}$ based on Clustering Probability Model (CPM). The $\mathrm{CH}$ node aggregates and forwards the data packets to the parent $\mathrm{CH}$ node in the DODAG. Yiju n et al. [23] proposed a Sink Oriented Layered Clustering (SLOC) protocol for WSN. The SLOC protocol divides the network space into rings of equal width. It performs intra-ring clustering and inter-cluster routing process to transfer the data from participant node to DODAG root.

Zhao, Ivan and Peter [26] proposed a region based routing protocol (ER-RPL). It improves the energy efficiency and packet delivery ratio in LLN. It 
divides the network space into several regions. It sets the reference node randomly in each region and it establishes the point-to-point best route in the region. Finally, it aggregates data from the whole region and transfers the data to the DODAG root. $\mathrm{Zh}$ a o, Peter and Henry [27] proposed a hybrid cluster parent routing protocol (HECRPL). It enhances the reliability of the nodes and increases the energy efficiency by Cluster Parent Selection (CPS), overhearing coordination mechanism to avoid the duplicate data, loss recovery scheme for the lost data packet and refined the transmission power.

Jung, Jong-Yong and Hwa-Young [7] proposed a fuzzy based energy efficient cluster routing protocol (FEMCHRP) for WSN. It selects the $\mathrm{CH}$ node based on the routing metrics, battery level, data frequency and node density to transfer the data. $\mathrm{R}$ a $\mathrm{n}$ a et al. [14] proposed a multiple cluster head selection routing protocols for WSN. The Cluster Members (CM) forward the data to the $\mathrm{CH}$ node. The Cluster Head Leader (CHL) node collects the data from the $\mathrm{CH}$ node and it forwards the data to the Base Station (BS). Nay a k and A nurag [11] proposed a fuzzy cluster routing protocol, to prolong the network lifetime in WSN. It follows the LEACH behaviour, to select the $\mathrm{CH}$ node. It maintains two levels of nodes between the source node and the sink node namely, SuperCluster Head $(\mathrm{SCH})$ and Cluster Head $(\mathrm{CH})$. The fuzzy descriptor is applied to the $\mathrm{CH}$ node and it elects the $\mathrm{SCH}$ node among the $\mathrm{CH}$ node for transferring the data to the mobile sink.

Gaddour, Anis and Mohamed [4] proposed fuzzy-based Objective Functions (OF-FL), which take into account the routing metrics, ETX, delay, hop count and Link Quality Level (LQL), to provide the QoS in LLN. A 1 jarrah [2] proposed a multi fuzzy logic model based objective function (ML-FL) for LLN. It considers the node metrics (node energy, ETX and neighbours in connectivity), channel metrics (channel capacity, RERQ and channel bandwidth) and link oriented metrics (link stability, hop count and mobility), to extend the network lifetime. K a m g u e u et al. [8] proposed a fuzzy logic based RPL protocol for LLN. It applies the fuzzy logic over the routing metrics, delay, ETX and energy, to transfer the data efficiently.

\section{System model}

\subsection{Network model}

The network area contains $N$ number of sensor nodes, which are deployed statically and distributed uniformly. It has one sink or DODAG root, which is located in the center of the network area. All the nodes are deployed in a circular area of radius $R$ and have the same initial energy [19].

Fig. 1 shows that the network area is divided into rings of equal width, by computing the finest ring width $r$ for the network area. In the intra-ring clustering process, it forms clusters and selects the Cluster Head $(\mathrm{CH})$. In inter-cluster routing, the $\mathrm{CH}$ selects the optimum parent $\mathrm{CH}$ node in the upward layer, considering the routing metrics namely, ETX and RER. 


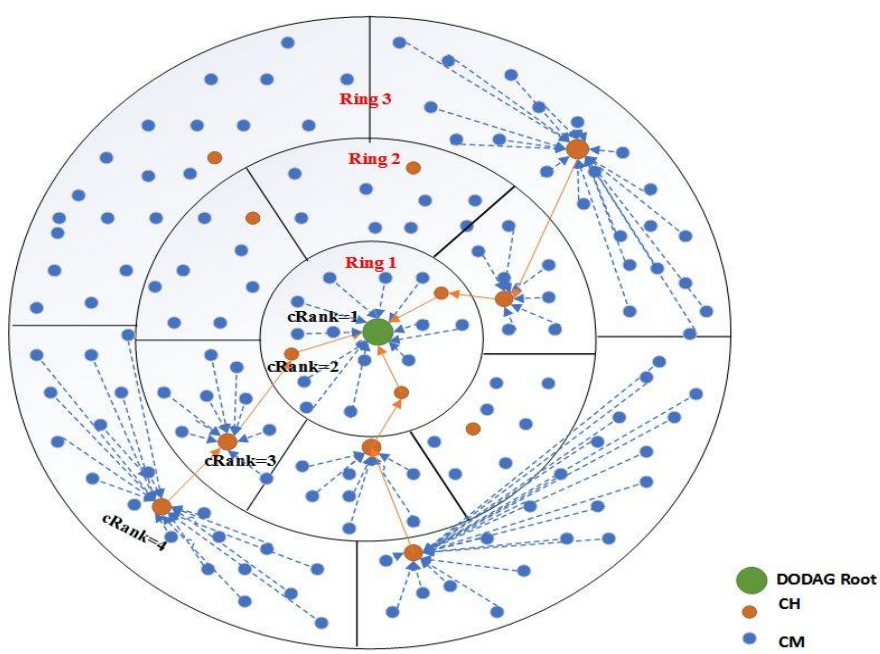

Fig. 1. Ring and cluster formation

\subsection{Energy model}

Energy model considers the factors, namely sensing, transmission, reception and aggregation for the energy consumption. The energy consumed for sensing, transmitting, receiving and aggregating one bit of data packet is represented as $e_{s}, e_{t}$, $e_{r}$ and $e_{a}$, respectively. The energy consumed for transmission of one bit over a distance of (dist) is computed as

$$
e_{t}(\text { dist })=\varepsilon_{1}+\varepsilon_{2} \times \text { dist }^{\alpha},
$$

where $\varepsilon_{1}$ is electronic transmission energy consumption, $\alpha$ is decay exponent and $\varepsilon_{2}$ is the amplifier energy. It is considered the variable transmission power level and it follows the simplified radio channel model.

\section{The MCEA-RPL protocol design}

The MCEA-RPL protocol is designed to enhance the network lifetime in LLN. It consists of three phases, namely, ring creation, intra ring clustering process and the intercluster routing process. Initially, it creates the virtual rings and the sensor nodes are distributed randomly in the network area. The ring width is fixed based on the energy consumption of nodes and the network area. The intra-ring clustering process performs two operations, namely cluster formation and $\mathrm{CH}$ selection. The cluster formation is based on the energy consumption of nodes in each ring. The cluster is of smaller size closer to the sink node and its size increases gradually as it gets far away from the sink node. The inter-cluster routing applies the fuzzy logic over ETX and RER to select the best $\mathrm{CH}$ parent node, for data transfer from participant node to DODAG root.

\subsection{Network ring creation}

The network area is divided into rings of equal width [23]. The number of rings $Q$ created in the network area with radius $R$, of ring width $r$, is given by 


$$
Q=\frac{R}{r}
$$

The sensor nodes are uniformly distributed and the number of sensor nodes present in each ring $n_{q}$, is

$$
n_{q}=\frac{(2 q-1) r^{2}}{R^{2}} \times N
$$

where $q=1,2, \ldots, Q$.

The innermost ring is considered as a single cluster. It directly forwards its data to the sink. Moreover, some of the nodes acts as cluster heads, which receive and forward the data packet from the downward layer nodes to the sink. is

The energy consumption of the cluster member $E_{1, \mathrm{CM}}$, in the innermost ring $q_{1}$

$$
E_{1, \mathrm{CM}}=e_{s}+e_{t} \times \mathrm{dist},
$$

where $e_{s}, e_{t}$ represents the energy consumption for data sensing and transmission, respectively.

The energy consumption of the cluster head $E_{1, \mathrm{CH}}$, in the innermost ring $q_{1}$ is

$$
E_{1, \mathrm{CH}}=\left(e_{s}+e_{t} \times \operatorname{dist}\right)+\frac{\sum_{q=2}^{Q} \rho n_{q}}{j_{q}}\left(e_{r}+\left(e_{t} \times \text { dist }\right)\right),
$$

where dist indicates distance between sensor node in the innermost ring and the sink node, which is calculated by

$$
\operatorname{dist}=\int_{\theta=0}^{2 \Pi} \int_{b=0}^{r} b \lambda_{1} b \partial b \partial \theta=\frac{2}{3} r
$$

where $b$ denotes the area of the ring and $\theta$ denotes the angle of the ring.

The $\mathrm{CH}$ role is supposed to be rotated equally in the inner most ring. If a node acts as a cluster head $\mathrm{CH}$ in a particular round, it will not act as $\mathrm{CH}$ node for the next $\frac{n_{1}}{j_{1}}-1$ rounds. The general aggregation model is applied in the downward layers [11]. The total energy consumption $E_{1}$ in the innermost ring is computed by

$$
\begin{gathered}
E_{1}=\frac{\left(\frac{n_{1}}{j_{1}}-1\right) \times E_{1, \mathrm{CM}}+E_{1, \mathrm{CH}}}{\left(\frac{n_{1}}{j_{1}}-1\right)}=e_{s}+\left(e_{t} \times \mathrm{dist}\right)+\frac{\sum_{q=2}^{Q} \rho n_{q}}{j_{q}}\left(e_{r}+\left(e_{t} \times \mathrm{dist}\right)\right)= \\
=e_{s}+\varepsilon_{1}+\varepsilon_{2} \times\left(\frac{2}{3} r\right)^{\alpha}+\rho \times \frac{R^{2}-r^{2}}{r^{2}} \times\left[e_{r}+\varepsilon_{1}+\varepsilon_{2} \times\left(\frac{2}{3} r\right)^{\alpha},\right.
\end{gathered}
$$

where $\varepsilon_{1}, \varepsilon_{2}, r$ represents the electronic energy consumption, amplifier energy and ring width, respectively.

To simplify the average energy consumption $E_{1}$ of the inner most rings, we differentiate the (7) with respect to $r$ and we construct the Quadratic equation, represented as 


$$
\frac{\partial E_{1}}{\partial r}=0 \Rightarrow X r^{\alpha+2}+Y r^{\alpha}+Z=0,
$$

where $X, Y$ and $Z$ are obtained by:

$$
\begin{gathered}
X=\left(\frac{2}{3}\right)^{\alpha} \times \varepsilon_{2} \times \alpha \times(1-\rho), \\
Y=\left(\frac{2}{3}\right)^{\alpha} \times \varepsilon_{2} \times \rho \times R^{2}(\alpha-2), \\
Z=2 \times \rho \times R^{2} \times\left(e_{r}+\varepsilon_{1}\right),
\end{gathered}
$$

where $\alpha, \rho$ represents exponent decay and aggregation ratio, respectively. Finally, the ring width $r$ is computed as follows:

$$
r=\left(\frac{Z}{X}\right)^{4}=\left(\frac{\rho \times\left(e_{r}+\varepsilon_{1}\right)}{\left(\frac{2}{3}\right)^{2} \times \varepsilon_{2} \times(1-\rho)}\right)^{\frac{1}{4}} \times \sqrt{R}=4.12 \sqrt{R} .
$$

The lifetime of a node is calculated as $E_{\text {init }} / E_{1}$ rounds, where $E_{\text {init }}$ represents the initial energy of a node. We decide the number of cluster heads $j_{1}$ in the innermost ring depends on the application requirement.

We compute the number of cluster head in the $q$-th ring $j_{q}$, where $q=2,3, \ldots, Q$, such that it balances the energy consumption in each ring [23]. The energy consumption for aggregating and transmitting a data packet by a cluster head in the $q$-th ring $E_{q, \mathrm{CH}}$, is given by

$$
\begin{aligned}
E_{q, \mathrm{CH}}=e_{s}+ & e_{r}\left(\frac{n_{q}}{j_{q}}-1\right)+\left(e_{a} \times \frac{n_{q}}{j_{q}}\right)+\left(\frac{\rho n_{q}}{j_{q}} \times e_{t} \times \operatorname{dist}_{q, q-1}\right)+ \\
& +\left(\frac{\sum_{i=q+1}^{Q} \rho n_{i}}{j_{q}} \times\left[e_{r}+e_{t} \times \text { dist }_{q, q-1}\right]\right),
\end{aligned}
$$

where $n_{q}, j_{q}$ represent the numbers of sensor nodes in $q$-th ring and number of $\mathrm{CH}$ in $q$-th ring, respectively. The dist ${ }_{q, q-1}$ indicates the distance between sensor nodes in $q$-th ring and $(q-1)$-th ring and is given by

$$
\operatorname{dist}_{q, q-1}=\varepsilon \times\left(\text { dist }_{q, 1}-\text { dist }_{q-1,1}\right), \quad \varepsilon \geq 1,
$$

where $\varepsilon$ is a constant and dist ${ }_{q, q-1}$ indicates the distance between the sensor node in $q$-th ring and the sink node. Thus, it fine-tunes the number of $\mathrm{CH}$ nodes in the inner most ring and it tries to balance the energy consumptions in all the rings.

\subsection{Intra-ring clustering}

The intra-ring clustering has two sub-processes namely, cluster formation and cluster head selection. 


\subsubsection{Cluster formation}

The number of $\mathrm{CH}$ in each ring is decided by the energy consumption in the innermost ring, i.e., $E_{q, \mathrm{CH}}=E_{1, \mathrm{CH}}$ where $q=2,3, \ldots ., Q$. In our simulation, we consider the number of nodes $N=150$, overall network region $W=150 \mathrm{~m}$, ring width $r=50$, number of $\operatorname{ring} Q=\frac{W}{r}=\frac{150}{50}=3$ and $\alpha=2$. The number of nodes in each ring $n_{q}$ are 15,50 and 85 , respectively. The number of sensor nodes in the innermost ring $n_{1}$ is 15. Initially, we pick the number of $\mathrm{CH}$ in the innermost ring $j_{1}$, varying from 7,5 , $4,3,2$ based on $1 / 2$ to $1 / 6$ of $n_{1}$ nodes. To balance the energy consumption of each ring, the MCEA-RPL forms the number of clusters in the rings nearby the sink and less number of clusters in the rings far away from the sink. The number of $\mathrm{CH}$ node $j_{n}$ is based on the energy consumption of each ring. It optimizes the number of $\mathrm{CH}$ in $q_{1}$ and it maintains the same energy consumption of sensor nodes in each ring. Finally, we obtain the optimal number of CH's are 3,6 and 4, respectively for the ring $q_{1}, q_{2}$ and $q_{3}$.

The area of each ring $A_{q}$ is calculated from the network area and represented by

$$
A_{q}=\Pi(q \times r)^{2}-\Pi((q-1) \times r)^{2},
$$

where $q=1,2, \ldots, Q$. The average area of cluster or cluster size $\operatorname{cs}_{q}$ is calculated from the area of rings and it is given by

$$
\mathrm{cs}_{q}=\frac{A_{q}}{j_{q}}, \quad q=1,2, \ldots, Q,
$$

where $A_{q}, j_{q}$ represent the area and the number of $\mathrm{CH}$ in $q$-th ring.

\subsubsection{Cluster head selection}

The cluster head selection has two phases, namely, setup phase and steady phase. During the setup phase, the node selects the $\mathrm{CH}$ stochastically in the cluster. This approach gives the equal chance to all nodes to act as a $\mathrm{CH}$ and maintains equal residual energy among the nodes within a cluster. Each node in the cluster generates the random number between 0 and 1 . The node with the largest threshold value is selected as the cluster head, for that time period called round:

$$
T(n)=\left\{\begin{array}{l}
\frac{1}{n_{q, j}} \times\left(\text { round } / n_{q, j}\right) \quad \forall n \in G, \\
0 \quad \forall n \notin G,
\end{array}\right.
$$

where $n_{q, j}$ is the total number of nodes in $q$-th ring of $j$-th cluster and $G$ is the set of nodes that do not act as a cluster head in last $\frac{1}{n_{q, j}}$ rounds.

\footnotetext{
Algorithm 1: Intra-Ring Clustering Algorithm

1: Input:

2: Number of ring $Q$, Area of ring $A_{q}$, Number of nodes in each ring $N_{q}$

3: Output:

4: Number of $\mathrm{CH}$ in each ring $j_{q}$
} 


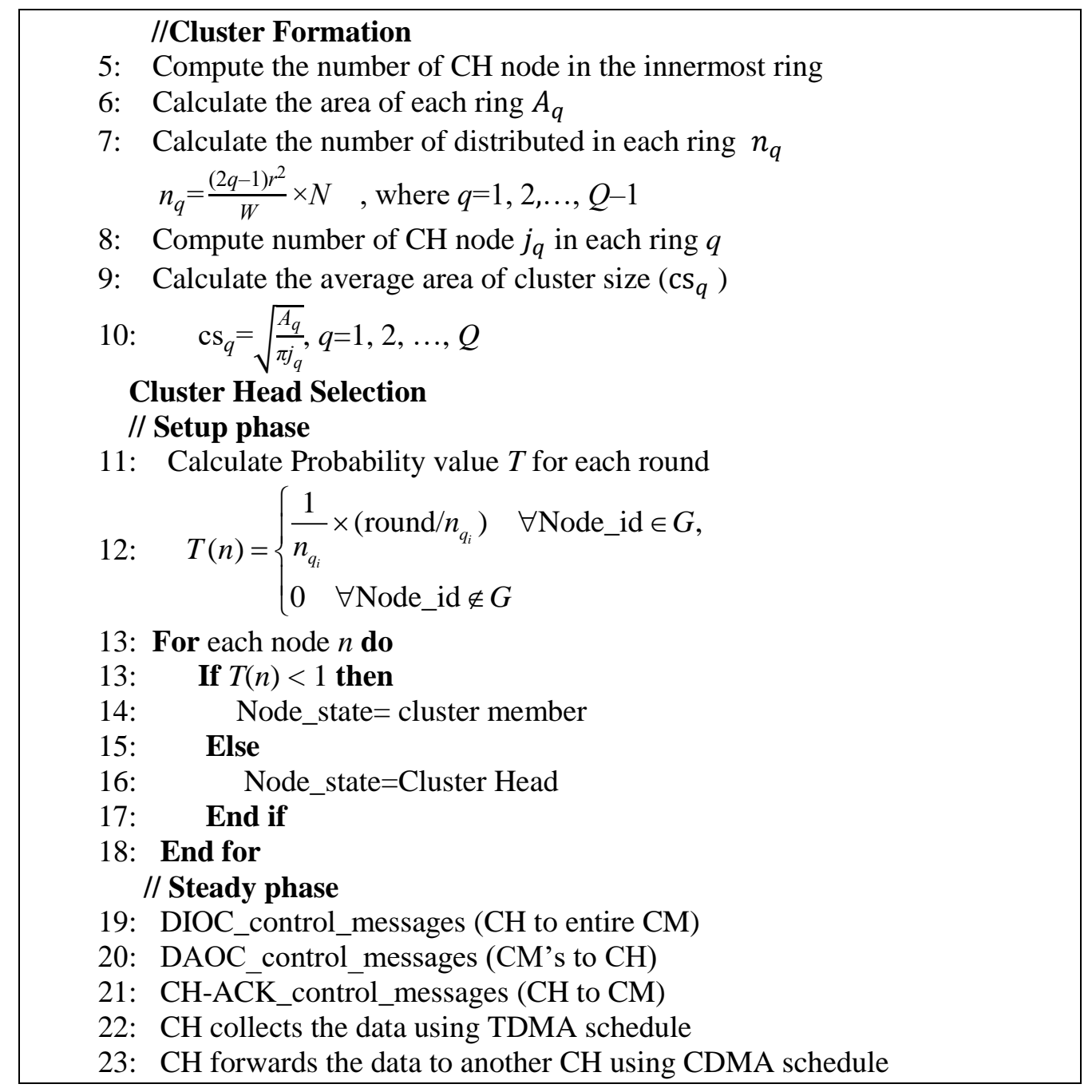

In steady phase, $\mathrm{CH}$ node multicasts the control message DODAG Information Object for Cluster (DIOC) to the entire CM. The CM replies with the control message DODAG Advertisement Object for Cluster (DAOC) to $\mathrm{CH}$ node. The $\mathrm{CH}$ node sends the Cluster Head ACKnowledgement (CH-ACK) message to the entire CM. The CM sends data to the $\mathrm{CH}$ node using the TDMA schedule. The $\mathrm{CH}$ node applies the data aggregation $(\rho=0.2)$, to aggregate data from $\mathrm{CM}$. The $\mathrm{CH}$ node forwards the data to the parent node or DODAG root using the CDMA time schedule.

\subsection{Inter cluster routing}

Inter cluster routing maintains the DODAG topology, to select the best parent node for data transfer. It implements the route establishment process of cluster based RPL [20]. The CH node broadcast the DIOC control messages to all CM inside the cluster. The CM node sends the DAOC control messages to $\mathrm{CH}$ node within the trickle time. Finally, the $\mathrm{CH}$ node sends the $\mathrm{CH}-\mathrm{ACK}$ messages to the cluster members and 
updates the node information in the routing table. During the routing process, it maintains the $\mathrm{CH}$ node information in two states namely, original optimal parent and suboptimal parent. The suboptimal parent collects and aggregates the data from the cluster members. The parent node passes its information to the participant node in the option field (DAG metric container) of the DIOC control message. We apply the fuzzy logic on the routing metrics ETX and RER, to select the optimal parent node, for data transfer. The Suboptimal node selects the best original parent among the preferred original parent node using cRank. The suboptimal parent forwards the aggregated data to the parent $\mathrm{CH}$ node or optimal parent node. The original optimal parent gathers the data packets from suboptimal parent and it forwards the data packet to DODAG root or parent $\mathrm{CH}$ node without data aggregation.

\subsubsection{Fuzzy inference system}

Fuzzy inference system (FIS) is a type of input and output mapping system applying fuzzy logic. In FIS, the major important components are fuzzification, inference engine and defuzzification $[10,12]$.

- Fuzzification

Fuzzification is a process of converting the crisp value into fuzzy set value. In MCEA-RPL, RER and ETX are the input variables to the fuzzy inference system.

Linguistic variable: The Linguistic variable belongs to the fuzzy set and its values are words or sentences rather than numbers. In MCEA-RPL, the first fuzzy input variable RER contains three linguistic variables namely, low, medium and high. The second fuzzy input variable ETX contains three linguistic variables namely, short, average and long. Moreover, the output variable quality of $\mathrm{CH}$ parent node contains five linguistic variables namely, awful, bad, good, very good and excellent.

Membership Function: Membership functions are used to evaluate the linguistic variable. Fig. 2-4 shows that the membership functions of input and output fuzzy variable. Here, we have chosen the trapezoidal and triangle membership function for selecting the best $\mathrm{CH}$ node. The membership function value is a universe of discourse and its ranges between 0 and 1 .

The triangle membership function generation representation is given by

$$
\mu_{A_{1}}(y)=\left\{\begin{array}{l}
0, \quad z \leq d_{1}, \\
\frac{z-d_{1}}{e_{1}-d_{1}}, \quad d_{1} \leq z \leq e_{1}, \\
\frac{f_{1}-z}{f_{1}-e_{1}}, \quad e_{1} \leq z \leq f_{1}, \\
0, \quad f_{1} \leq z .
\end{array}\right.
$$

The trapezoidal membership function generation representation is given 


$$
\mu_{A_{2}}(y)=\left\{\begin{array}{l}
0, \quad z \leq d_{2}, \\
\frac{z-d_{2}}{e_{2}-d_{2}}, \quad e_{2} \leq z \leq f_{2}, \\
1, \quad e_{2} \leq z \leq f_{2}, \\
\frac{f_{1}-z}{f_{1}-e_{1}}, \quad f_{2} \leq z \leq g_{2}, \\
0, \quad g_{2} \leq z .
\end{array}\right.
$$

The membership function of the first fuzzy input variable RER is represented in Fig. 2 [9]. The variable low and high are resented using the trapezoidal shape of the membership and the variable medium is represented using triangle shape membership function.

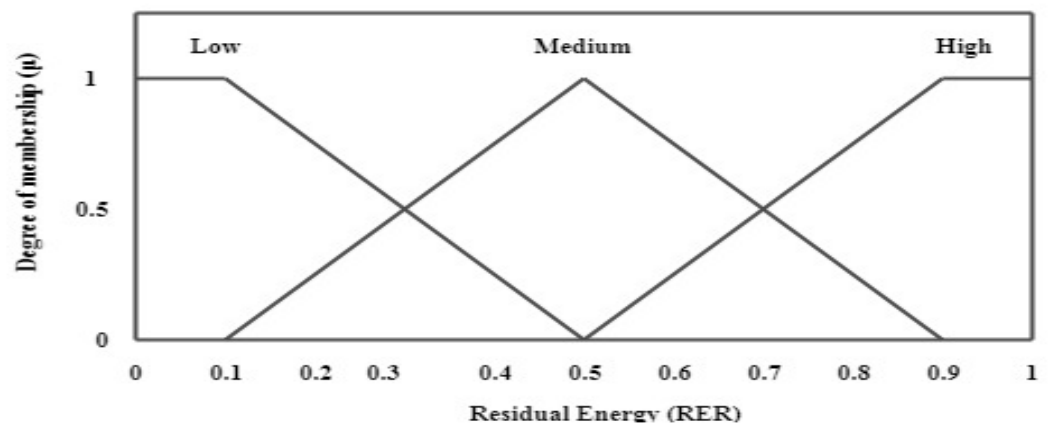

Fig. 2. Membership function of residual energy

The membership function of second fuzzy input variable ETX is described in Fig. 3 [22]. The linguistic variables short and long are represented using the trapezoidal shape of the membership and the variable average is represented using triangle shape of the membership function.

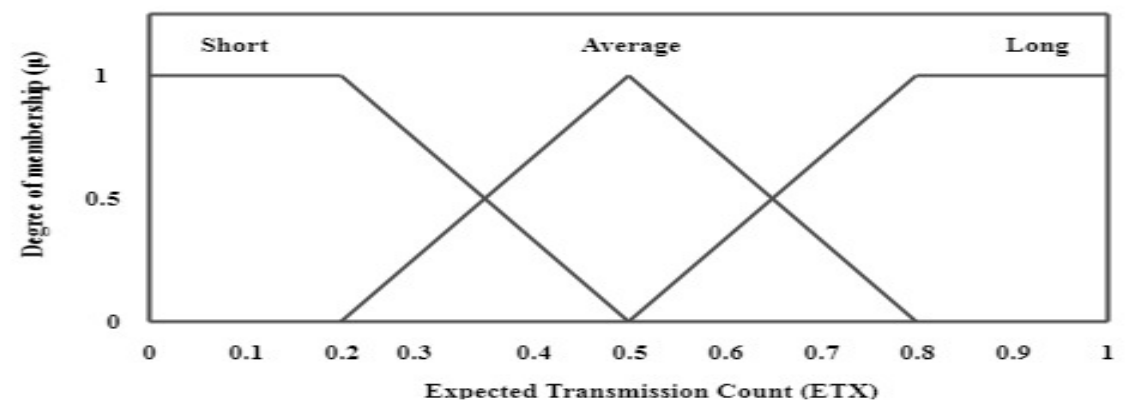

Fig. 3. Membership function of expected transmission count

The membership function of output fuzzy variable quality of $\mathrm{CH}$ node is represented in Fig. 4 [9]. 


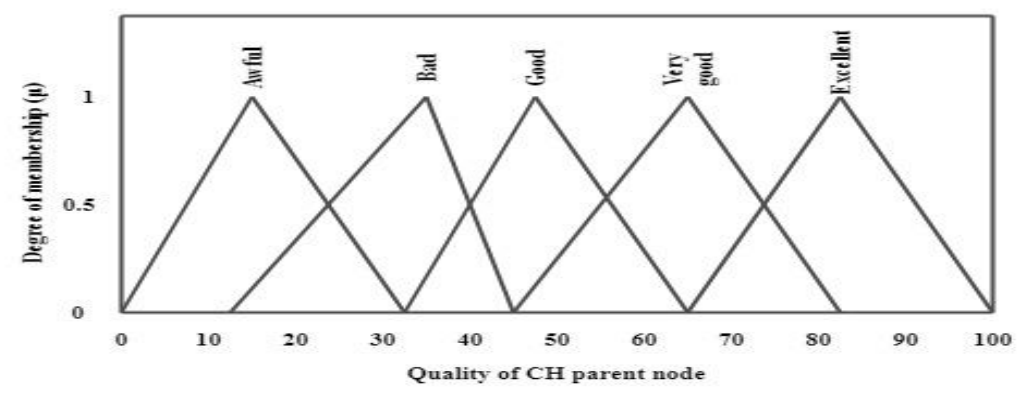

Fig. 4. Membership function of the quality of $\mathrm{CH}$ parent node

- Fuzzy inference rule

In MCEA-RPL, we use the routing metrics ETX and RER, to select the best route, for transferring the data from source to DODAG root following the upward routing. The fuzzy rule contains 2 input controllers and 3 membership functions which are represented as $3^{2}=9$ rules. The fuzzy rules are given in Table 1 .The quality of parent $\mathrm{CH}$ node ranges between 0 and 100 . The fuzzy rules are described using "if-then" rules and it is evaluated by the Mamdani model [13]. It varies based on the application requirements.

Table 1. Fuzzy rules
\begin{tabular}{|c|c|c|c|}
\hline No & RER & ETX & Quality of CH node \\
\hline 1 & low & short & good \\
\hline 2 & low & average & bad \\
\hline 3 & low & long & awful \\
\hline 4 & medium & short & very good \\
\hline 5 & medium & average & good \\
\hline 6 & medium & long & bad \\
\hline 7 & high & short & excellent \\
\hline 8 & high & average & very good \\
\hline 9 & high & long & good \\
\hline
\end{tabular}

- Defuzzification: It is a process of getting the crisp value from fuzzy set value. In MCEA-RPL, we have used the Center Of Area (COA) method for the defuzzification process [15]. The center of area is given by

$$
\operatorname{COA}(z)=\frac{\int \mu_{A}(z) y d z}{\int \mu_{A}(z) d z},
$$

where $\mu_{A}(z)$ is the aggregated output membership function for different input variables, $\operatorname{COA}(z)$ is defuzzified fuzzy output variable.

\subsection{2. cRank calculation process}

The cRank represents the number of $\mathrm{CH}$ nodes between DODAG root and suboptimal parent node. The rank increase value is calculated from step value and min_hop_rank_increase_value. The default value of min hop rank increase is 256 [20]. The step_value is calculated from defuzzified value of routing metrics RER and ETX. The suboptimal parent rank cRank $(N)$ is given by: 
$c \operatorname{Rank}(N)=c \operatorname{Rank}(P N)+\operatorname{Rank} \_$increase,

Rank_increase $=$ step_value + Min_Hop_Rank_Increase .

The inter-cluster routing algorithm is represented, for transferring the data from the downward layer to DODAG root and it is given in below.

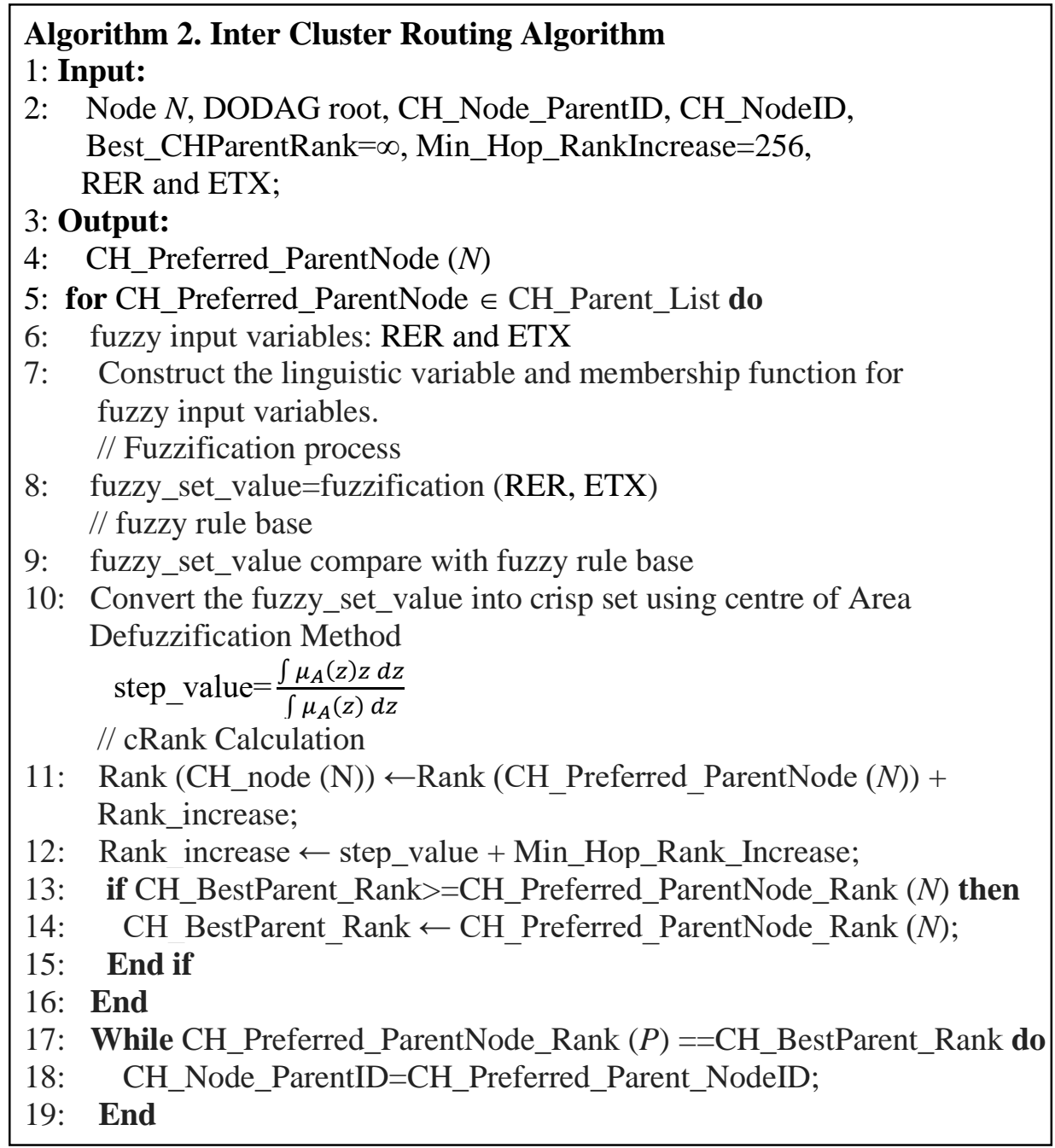

\section{Result and discussions}

\subsection{Simulation setting and parameters}

The objective of the simulation is to show, how the MCEA-RPL protocol is better than RPL and IRPL. The COOJA simulator is used to conduct the simulation [18]. We use sky mote in our simulation. $150 \mathrm{RPL}$ routers and 1 DODAG root are taken for simulation. The overall network radius is $150 \mathrm{~m}$. The simulation is conducted in two scenarios, a. Transmitting one packet per minute and $\mathbf{b}$. Transmitting six packets 
per minute. We set the DIOC time interval as $(12 \mathrm{~ms} *$ the number of $\mathrm{CM})$. The simulation parameter details are given in Table-2.

\begin{tabular}{|c|c|}
\hline Parameter & Value \\
\hline Operating system & Contiki 2.7 \\
\hline Routing protocol & RPL \\
\hline Node type & Sky mote \\
\hline Radio environment & Unit Disk Graph Medium (UDGM) \\
\hline Simulation hour & $1 \mathrm{~h}$ \\
\hline MAC layer & ContikiMAC/6LowPAN \\
\hline Network over all radius & $150 \mathrm{~m}$ \\
\hline Number of nodes & 150 RPL Router + 1 DODAG root \\
\hline Transmission range & $100 \mathrm{~m}$ \\
\hline Data packet timer & $60 \mathrm{~s}$ \\
\hline RPL parameter & MinHopRankIncrease $=256$ \\
\hline$e_{S}($ data sensing energy) & $5 \mathrm{~nJ}$ per 1 bit \\
\hline$e_{r}($ rx receiving energy rate $)$ & $50 \mathrm{~nJ}$ per 1 bit \\
\hline$e_{a}($ data aggregation energy $)$ & $5 \mathrm{~nJ}$ per 1 bit per 1 signal \\
\hline$\varepsilon$ (data approximation ratio) & 1.1 \\
\hline$\varepsilon 1$ (tx transmission energy rate) & $50 \mathrm{~nJ}$ per 1bit \\
\hline$E_{2}(\mathrm{tx}$ amplifier energy rate) & $100(\mathrm{pJ} . \mathrm{bit}) / \mathrm{m}^{2}$ \\
\hline $\mathrm{CH}$ changes & 1000 bits per 1 round \\
\hline$\alpha$ (energy decay exponent) & 2 \\
\hline Initial energy $\left(E_{\text {init }}\right)$ & $2 \mathrm{~J}$ \\
\hline$\rho$ (data aggregation rate) & 0.2 \\
\hline
\end{tabular}

\subsection{Performance evaluation results}

We simulate and compare the performance of MCEA-RPL with RPL and IRPL in the above mentioned scenario.

\subsubsection{Average packet loss ratio}

The poor route selection and increased number of hops between source and destination lead to increased packet loss. We simulate with the packet rate of one and six packets per minute. It shows the packet loss increases as the network size increases.

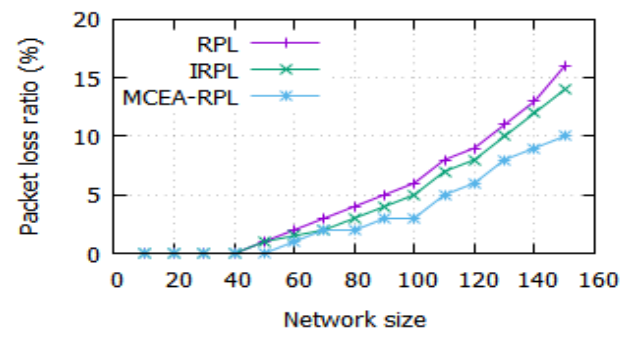

Fig. 5. Packet loss ratio vs. network size

Fig. 5 shows that packet loss rate on transfer of one packet per minute. It is observed that the packet loss ratio is lesser in MCEA-RPL compared to IRPL and RPL. In MCEA-RPL, as most of the nodes are located in the coverage area of 
neighbour layer and the application of fuzzy logic over the routing metrics ETX and RER, for route selection, results in lower packet loss rate. Fig. 6 shows the packet loss rate on the transfer of six packets per minute. It is noted that the packet loss increases, as the data transfer rate increases. It is observed that the packet loss rate is lesser in MCEA-RPL compared to IRPL and RPL.

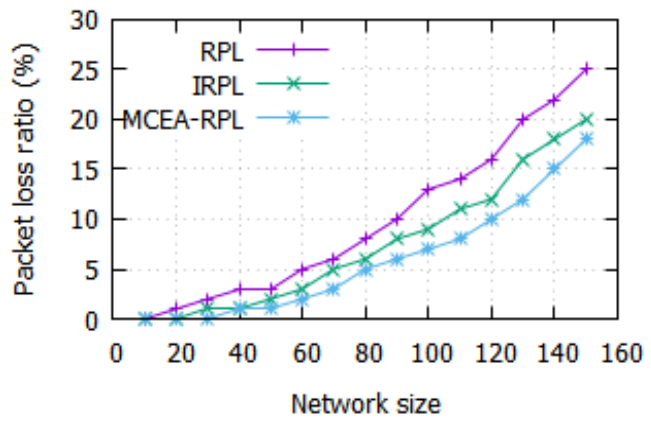

Fig. 6. Packet loss ratio vs. Network size

\subsubsection{Number of parent changes}

The number of parent change indicates the network stability. The stable networks have less number of topology changes during the data transmission. The simulation is done for one hour. Fig. 7 shows that the average parent change values of MCEA-RPL, IRPL and RPL. The parent change values of MCEA-RPL, IRPL and RPL are $0.15,0.18$ and 0.28 , respectively. It is observed that MCEA-RPL forms the stable network, resulting in an extended network lifetime, compared to IRPL and RPL.

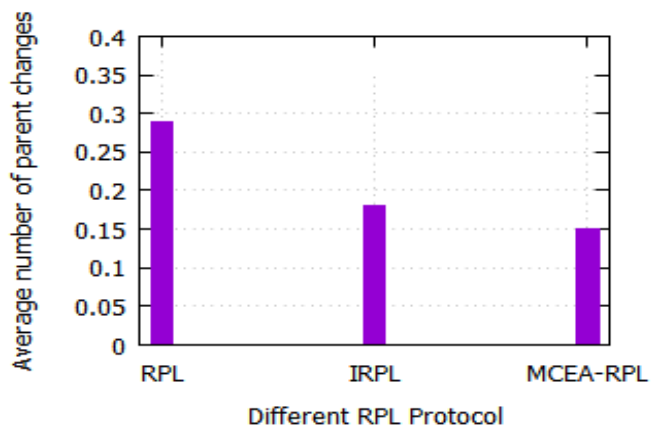

Fig. 7. Number of parent changes

\subsubsection{Average end-to-end delay}

Fig. 8 shows that average end-to-end delay (latency) of RPL, IRPL and MCEA-RPL. The average delay of MCEA-RPL, IRPL and RPL are $0.8 \mathrm{~ms}, 1.1 \mathrm{~ms}$ and $1.3 \mathrm{~ms}$, respectively. It is noted that the average end-to-end delay is below $1.3 \mathrm{~s}$ for 6 hops. MCEA- RPL has lower delay compared to RPL and IRPL, due to reduced node failures and route breakages during data transfer. It is achieved by selecting the optimal parent for forwarding the data to the sink. 


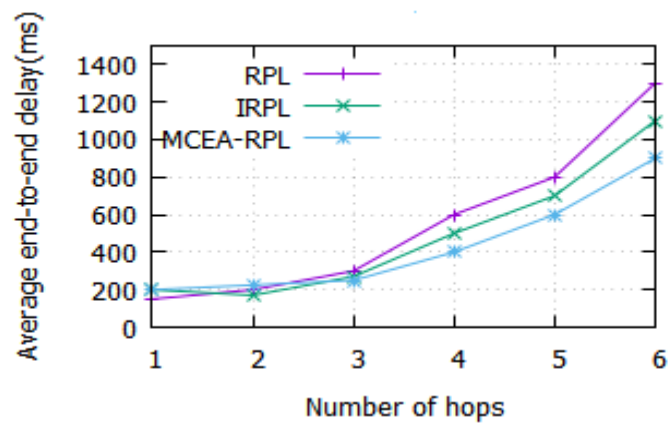

Fig. 8. Average end-to-end delay vs. number of hops in the network

\subsubsection{Average packet loss ratio due to node failure}

We simulate with the packet rate of one and six packets per minute. Fig. 9 shows that the packet loss rate due to node failure with the packet rate of one packet per minute. We calculate the packet loss ratio for the amount of failure nodes ranging from 0 to 60. The average packet loss ratio of MCEA-RPL, IRPL and RPL are $20 \%, 23 \%$ and $30 \%$, respectively for the failed node size is 60 . It shows that packet loss rate increases, as the number of failure node increases. MCEA-RPL can find the alternate original optimal parent quickly, which results in reduced packet loss compared to IRPL and RPL.

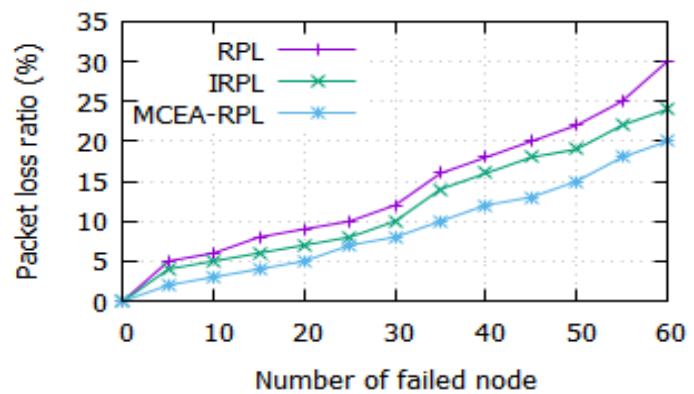

Fig. 9. Average packet loss ratio vs. number of failed nodes

Fig. 10 shows the packet loss rate due to node failure with the packet rate of six packets per minute. We observed that the packet loss ratio of MCEA-RPL, IRPL and RPL are $28 \%, 32 \%$ and $40 \%$, respectively for the failed node size is 60 .

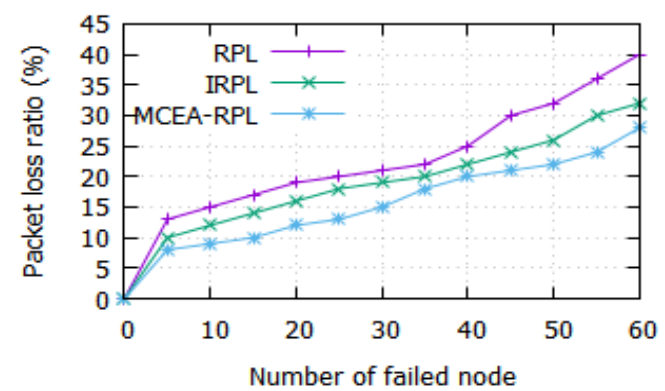

Fig. 10. Average packet loss ratio vs. number of failed nodes 


\subsubsection{Average power consumption of network nodes}

Fig. 11 shows the power consumption of nodes with the packet rate of one packet per minute. It is observed that the amount of power consumption increases, as the number of node increases in the network. It is noted that MCEA-RPL consumes less energy than IRPL and RPL, as its CH node transmits the aggregated data packets to the sink node through the optimal parent. The average power consumptions of MCEA-RPL, IRPL and RPL are $5.2 \mathrm{~mW}, 6.5 \mathrm{~mW}$ and $7 \mathrm{~mW}$, respectively for the network size of $150 \mathrm{~m}$.

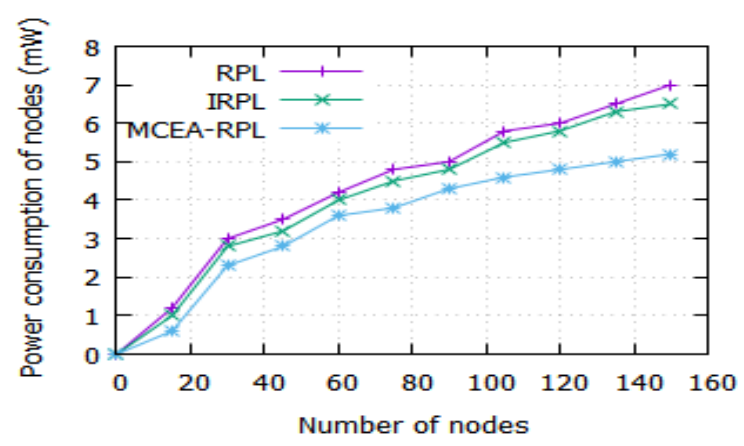

Fig. 11. Average power consumption vs. number of nodes

Fig. 12 shows the power consumption of nodes with the packet rate of six packets per minutes. The packet drop increases, as the amount of transfer rate increases in the network. The average power consumptions of MCEA-RPL, IRPL and RPL are $7 \mathrm{~mW}, 8.8 \mathrm{~mW}$ and $9 \mathrm{~mW}$, respectively for the network size of $150 \mathrm{~m}$.

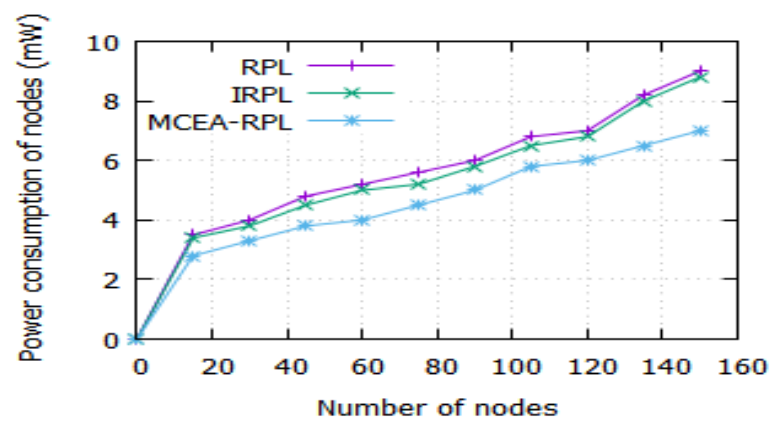

Fig. 12. Average power consumption vs. number of nodes

\section{Conclusion}

In this paper, we proposed a Multi-layer Cluster based Energy Aware Routing Protocol (MCEA-RPL) for LLN. It divides the network area into rings of equal width. It forms intra ring cluster to balance the energy consumption over the ring. The inter cluster routing selects the best optimal parent, by applying the fuzzy logic over the routing metrics ETX and RER. It reduces the path breakages due to early energy depletion on the nodes nearby the sink. The simulation result shows that MCEA-RPL 
has extended the network lifetime and increased the packet delivery ratio considerably.

As part of future work, MCEA-RPL is planned to introduce mobility in the network scenario and also to deploy it in the real-time environment.

\section{References}

1. A 1 b i s h i, S., B. S o h, B. A. U 11 a h, A. F. A 1 g a r n i. Challenges and Solutions for Applications and Technologies in the Internet of Things. - Procedia Computer Science, Vol. 124, 2017, pp. 608-614.

2. A 1 j a r r a h, E. Deployment of Multi-Fuzzy Model Based Routing in RPL to Support Efficient IoT. - International Journal of Communication Networks and Information Security (IJCNIS), Vol. 9, 2017, No 3, pp. 457-465.

3. G a d d o u r, O., K. A n i s. RPL in a Nutshell: A Survey. - Computer Networks, Vol. 56, 2012, No 14, pp. 3163-3178.

4. Gaddour, O., K. An is, A. Mohamed. Quality-of-Service Aware Routing for Static and Mobile IPv6-Based Low-Power and Lossy Sensor Networks Using RPL. - Ad Hoc Networks, Vol. 33, 2015, pp. 233-256.

5. He in ze l man, W. B., P. A. Ch and rak a s a n, B. Hari. An Application-Specific Protocol Architecture for Wireless Microsensor Networks. - IEEE Transactions on Wireless Communications, Vol. 1, 2002, No 4, pp. 660-670.

6. H u i, L., C. M i n. Research on the Distribution System Simulation of Large Company's Logistics under Internet of Things Based on Traveling Salesman Problem Solution. - Cybernetics and Information Technologies, Vol. 16, 2016, No 5, pp. 78-87.

7. J u n g, K., L. Jo n g-Y o n g, J. H w a-Y o ung. Improving Adaptive Cluster Head Selection of Teen Protocol Using Fuzzy Logic for WMSN. - Multimedia Tools and Applications, Vol. 76, 2017, No 17, pp. 18175-18190.

8. K a m g u e u, P. O., N. E m m a nu e 1, D. Th o m a s, F. O li vi e r. Fuzzy-Based Routing Metrics Combination for RPL. 2014.

Https://hal.inria.fr/hal-01093965/document

9. Logambigai, R., K. Arputharaj. Fuzzy Logic Based Unequal Clustering for Wireless Sensor Networks. - Wireless Networks, Vol. 22, 2016, No 3, pp. 945-957.

10. M o k h t a r, S., I. W a n, H. W a n, M. N. Norit a. Modeling Reservoir Water Release Decision Using Adaptive Neuro Fuzzy Inference System. - Journal of Information \& Communication Technology, Vol. 15, 2016, No 2.

11. N a y a k, P., D. A n u r a g. A Fuzzy Logic-Based Clustering Algorithm for WSN to Extend the Network Lifetime. - IEEE Sensors Journal, Vol. 16, 2016, No 1, pp. 137-144.

12. O th m a n, M., N. F. A. S it i. Deseasonalised Forecasting Model of Rainfall Distribution Using Fuzzy Time Series. - Journal of Information \& Communication Technology, Vol. 15, 2016, No 2 .

13. Pourjavad, E., V. R. Rene. A Comparative Study and Measuring Performance of Manufacturing Systems with Mamdani Fuzzy Inference System. - Journal of Intelligent Manufacturing, 2017, pp. 1-13.

14. R a n a, S., N. B. A 1 i, I. N a z r u l, I. J o h i r u l. Fuzzy Based Energy Efficient Multiple Cluster Head Selection Routing Protocol for Wireless Sensor Networks. - International Journal of Computer Network and Information Security, Vol. 7, 2015, No 4, p. 54.

15. Runkle r, T. A. Selection of Appropriate Defuzzification Methods Using Application Specific Properties. - IEEE Transactions on Fuzzy Systems, Vol. 5, 1997, No 1, pp. 72-79.

16. S a n k a r, S., P. S r i n i v a s a n. Internet of Things (Iot): A Survey on Empowering Technologies, Research Opportunities and Applications. - International Journal of Pharmacy and Technology, Vol. 8, 2016, No 4, pp. 26117-26141.

17. S a n k a r., S., P. S r i n i v a s a n. Composite Metric Based Energy Efficient Routing Protocol for Internet of Things. - International Journal of Intelligent Engineering and Systems, Vol. 10, 2017, No 5, pp. 278-286. 
18. S h a h r a, E. Q., R. S. T a r e k, M. S. E 1 h a di. A Comparative Study of Range-Free and RangeBased Localization Protocols for Wireless Sensor Network Using COOJA Simulator. International Journal of Distributed Systems and Technologies (IJDST), Vol. 8, 2017, No 1, pp. 1-16.

19. S h u, T., K. M a r w a n, V. S a r m a. Power Balanced Coverage-Time Optimization for Clustered wireless Sensor Networks. - In: Proc. of 6th ACM International Symposium on Mobile Ad Hoc Networking and Computing, 2005, pp. 111-120.

20. T a n, Y. R. RPL-Based Clustering Routing Protocol. - Internet Engineering Task Force, InternetDraft Draft-Tan-Roll-Clustering-00, Accessed on September 2017.

Https://tools.ietf.org/html/draft-tan-roll-clustering-00

21. Tripath i, J., C. D. O. J a u d e li c e, V. J e a n-P h i li p p e. A Performance Evaluation Study of rpl: Routing Protocol for Low Power and Lossy Networks. - In: Proc. of 44th Annual Conference on Information Sciences and Systems (CISS), 2010, IEEE, pp. 1-6.

22. W i n t e r, T. RPL: IPv6 Routing Protocol for Low-Power and Lossy Networks. 2012. Https://tools.ietf.org/html/rfc6550

23. Y i ju n, M., W. B a n g, L. W e n y u, T. Y. La u re n c e. A Sink-Oriented Layered Clustering Protocol for Wireless Sensor Networks. - Mobile Networks and Applications, Vol. 18, 2013, No 5, pp. 639-650.

24. Zhang, W., L. Ling, H. Guangjie, Z. Lincong. E2HRC: An Energy-Efficient Heterogeneous Ring Clustering Routing Protocol for Wireless Sensor Networks. - IEEE Access, Vol. 5, 2017, pp. 1702-1713.

25. Z h ang, W., H. Gu ang ji e, F. Y ong xi n, L. J a i me. IRPL: An Energy Efficient Routing Protocol for Wireless Sensor Networks. - Journal of Systems Architecture, Vol. 75, 2017, pp. 35-49.

26. Z h a o, M., W. H. H. I v a n, P. H. J. Ch o n g. An Energy-Efficient Region-Based RPL Routing Protocol for Low-Power and Lossy Networks. - IEEE Internet of Things Journal, Vol. 3, 2016, No 6, pp. 1319-1333.

27. Z h a o, M., H. J. C. P e t e r, C. C. H e n r y. An Energy-Efficient and Cluster-Parent Based RPL with Power-Level Refinement for Low-Power and Lossy Networks. - Computer Communications, Vol. 104, 2017, pp. 17-33.

Received 04.05.2018; Second Version 10.08.2018; Accepted 15.08.2018 